\title{
Parotid and submandibular duct calculi in three successive generations of one family
}

\author{
K. Nigel Bullock \\ M.A., F.R.C.S. \\ Department of Urology, Addenbrooke's Hospital, Cambridge CB2 2QQ
}

\begin{abstract}
Summary
A case is reported of chronic calculous parotitis beginning in a 12-month-old child. Further questioning revealed a history of parotid calculi in her mother, and submandibular calculi in her maternal grandmother. The features which indicate the diagnosis of parotid calculous disease are discussed and, from a review of the literature, some observations are made on management.
\end{abstract}

\section{Introduction}

Salivary calculi are relatively uncommon, although the sub-clinical incidence may be considerably higher. Parotid calculi represent only a small proportion of all salivary calculi and are particularly rare in children, with only a handful of reported cases.

\section{Case report}

The patient first presented as an 18-month-old girl with a 6-month history of right parotid swelling, initially diagnosed as mumps. Despite antibiotics, symptoms continued intermittently, so her parents sought specialist help. A right parotid sialogram was requested but she discharged a small calculus from the right parotid duct before it could be performed. The subsequent sialogram showed only chronic sialadenitis and sialectasis with 'lakes' of contrast. She then had no further trouble until aged 12 years. A parotid sialogram at this time showed similar changes within the gland and a radiolucent calculus in the extra-glandular part of the parotid duct (Fig. 1). She was advised to 'milk' the right parotid region and she has had no further symptoms over the succeeding year.

The patient's mother stated that she herself had passed a parotid calculus spontaneously 11 years previously, when aged 31 years. A right parotid sialogram showed chronic sialadenitis and sialectasis with no evidence of duct obstruction. She, too, was advised on conservative treatment. Since that time, she has had one mild attack of parotitis.

The 67-year-old grandmother of the patient was reported to have had intermittent facial swelling and inflammation in the past. When aged 45 years, she had passed a small calculus from the right submandibular duct. Following a recurrence of symptoms, she underwent removal of a further submandibular duct calculus and, when 62 years old, excision of the right submandibular gland.

\section{Discussion}

Parotid calculi account for $15-20 \%$ of all salivary calculi. They are commonest in adults, although it has been suggested that a slightly younger age group predominates (Elmostehy, 1968). Parotid calculi are notoriously difficult to diagnose and may be passed unnoticed. Part of the difficulty in diagnosis is due to lack of awareness. Patey (1966) noted over a 13-year period that one-third of his patients with a diagnosis of 'chronic parotitis' had parotid calculi which were not detected clinically, particularly when the inflammation was unilateral.

Parotid calculi are difficult to demonstrate by plain radiography, although an intra-oral film may be helpful. Parotid sialography, however, is the only reliable way to diagnose calculous disease. As well as demonstrating duct calculi, the sialogram may show calcareous paste throughout the duct system or microcalculi within the gland. Ultrasound may be helpful in the localization of calculi within the gland, but is not readily available in most centres (Pickrell, Trought and Shearin, 1978).

The treatment of parotid calculi has invariably been surgical. Seward (1968) recommends an intraoral approach to the distal part of the duct, if possible; otherwise, a pre-auricular incision is necessary, either to shell out a stone from the duct or to perform a total parotidectomy. Radiotherapy has been recommended for adults, but some of the irradiation inevitably affects the opposite gland, leaving the patient with a dry mouth. In children, simple measures such as 'milking' the gland and chewing gum are helpful, since the disease often burns itself out by atrophy of the gland (Eddey, 1966).

True parotid calculi are rare in children, with only a handful of cases reported (Smith and Coon, 1934; Kaufman, 1968; Grzegorowski, 1975; Treheux 


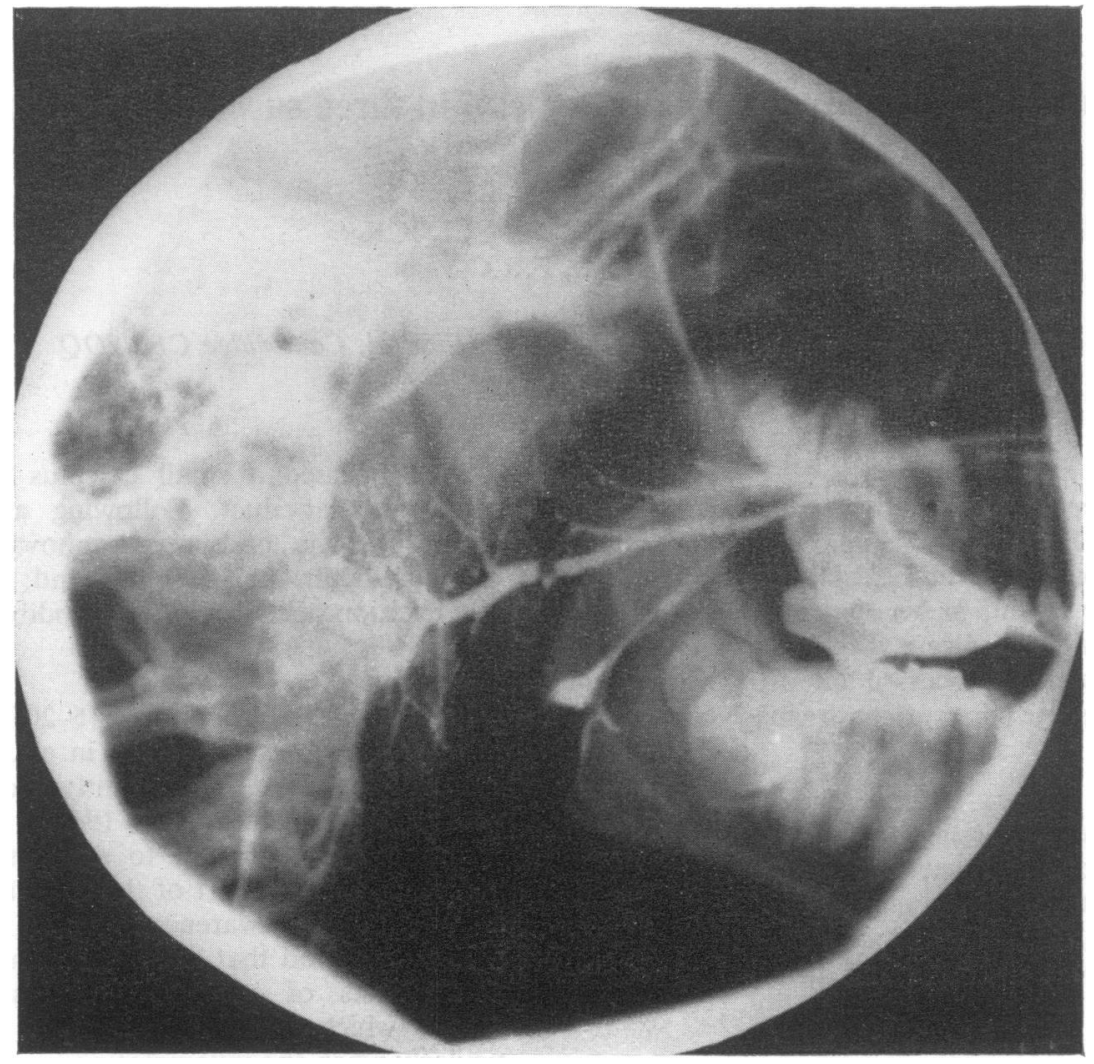

FIG. 1. Right parotid sialogram of the patient showing a filling defect (arrowed) in the parotid duct due to radiolucent calculus. $\stackrel{\circ}{\Phi}$

et al., 1975; Fesharaki et al., 1979). There have been no previous reports of true salivary calculi occurring in successive generations within one family. This situation must be distinguished from the commoner condition of punctate sialectasis with microcalculi which, histologically, is similar to benign lymphoepithelial disease; this disease process, which includes, at one extreme, Sjøgren's syndrome, is well recognized in children and does have a familial tendency (Katzen and Du Plessis, 1964; Suleiman, Thompson and Hobsley, 1979).

It may be, in this family, that there is an hereditary salivary abnormality predisposing to calculi or that there is a common environmental factor. It remains to be seen whether conservative treatment of calculous parotitis in this girl and her mother will be successful in averting the need for surgery.

\section{Acknowledgments}

I thank Mr W. G. Everett for his permission to report these cases which came under his care.

\section{References}

EDDEY, H.H. (1966) Recurrent parotitis and associated calculus, cyst or tumour. Medical Journal of Australia, 2, 581 .
Elmostehy, M.R. (1968) Parotid salivary calculus. Report of $\overrightarrow{\vec{A}}$ a case. Oral Surgery. Oral Medicine and Oral Pathology, $\frac{}{3}$ 26, 18.

FeshaRaki, R., Baloochy, M., Sonbolastan, M. \& Fotuhi, A. (1979) Parotid gland calculus. Report of a case. Journal of Laryngology and Otology, 93, 737.

GrzegorowsKI, M. (1975) Parotid calculus in a $3 \frac{1}{2}$-year-old 3 child. Otolaryngologia polska, 29, 205.

Katzen, M. \& Du Plessis, D.J. (1964) Recurrent parotitis in children. South African Medical Journal, 38, 122.

Kaufman, S. (1968) Parotid sialolithiasis in a child. American $ᄋ$ Journal of Diseases of Children, 115, 623.

Patey, D.H. (1966) The clinical features and diagnosis of $\mathrm{o}$ parotid calculi. Practitioner, 197, 67.

Pickrell, K.L., Trought, W.S. \& Shearin, J.C. (1978) The use of ultrasound to localize calculi within the parotid gland. Annals of Plastic Surgery, 1, 542.

SEWARD, G.R. (1968) Anatomic surgery for salivary calculi. $\stackrel{N}{\circ}$ Part VI. Calculi in the intraglandular part of the parotid $N$ gland. Oral Surgery, Oral Medicine, Oral Pathology, 26, 1.

SMITH, H.B. \& Coon, E.H. (1934) Salivary calculi with $\mathrm{\omega}^{\mathrm{\omega}}$ report of a case of stone in Stensen's duct in a boy aged $\sigma$ eleven years. Medical Times and Long Island Medical Journal, 62, 215.

Suleiman, S.I., Thompson, J.P.S. \& Hobsley, M. (1979) Recurrent unilateral swelling of the parotid gland. Gut, $2, \stackrel{\text { ? }}{?}$ 1102.

Treheux, A., Wayoff, M., Friot, J.C. \& Bretagne, M.C. (1975) Parotid lithiasis in children. Journal de Radiologie, $\overrightarrow{\mathbb{D}}$ d'Electrologie et de Médecine Nucléaire, 56, 167. 